\title{
Downregulated RPL6 inhibits lung cancer cell proliferation and migration and promotes cell apoptosis by regulating the AKT signaling pathway
}

\author{
Jin Zhang, Qianli Ma, Yu Han, Huanshun Wen, Zhenrong Zhang, Yang Hao, Fei Xiao, Chaoyang Liang \\ Department of Thoracic Surgery, China-Japan Friendship Hospital, Beijing, China \\ Contributions: (I) Conception and design: J Zhang, F Xiao; (II) Administrative support: C Liang; (III) Provision of study materials or patients: Q Ma, \\ Z Zhang; (IV) Collection and assembly of data: H Wen, Y Hao; (V) Data analysis and interpretation: Y Han; (VI) Manuscript writing: All authors; (VII) \\ Final approval of manuscript: All authors. \\ Correspondence to: Fei Xiao, MD; Chaoyang Liang, MD. Number 2, Yinghua East Street, Chaoyang District, Beijing 100029, China. \\ Email: shawbjmu@163.com; liangchaoyang@zryhyy.com.cn.
}

\begin{abstract}
Background: Lung cancer is still one of the most common causes of cancer-related mortality, and the overall survival is less than $5 \%$. It is important and necessary to elucidate the mechanism of lung cancer progression. Recently, more and more research has demonstrated that many ribosomal proteins (RPs) are dysregulated in tumors. Among these RPs, ribosomal protein L6 (RPL6) is reported to promote cell growth and cell cycle progression in gastric cancer cells through upregulating cyclin E. However, its function in lung cancer is still unknown.
\end{abstract}

Methods: We first explored RPL6 expression in lung cancer samples. Next, we used gene knockdown to analyze the unknown regulatory role of RPL6 in lung cancer carcinoma and lung cancer cell lines. Furthermore, we explored the potential signaling pathway of RPL6 with Western blotting.

Results: In this study, we demonstrated that RPL6 expression was highly expressed in lung cancer tissues and lung cancer cell lines. RPL6 downregulation inhibited H1299 and H2975 cell proliferation, migration and induced cell apoptosis. Also RPL6 downregulation increased the protein levels of cleaved caspase-3 and Bax, while decreasing the protein level of B-cell lymphoma 2 (Bcl-2). Western blotting results showed that proteins activating the AKT signaling pathway, such as p-AKT and p-S6, were downregulated in RPL6 knockdown lung cancer cells.

Conclusions: These findings show that RPL6 can be used as a potential therapeutic and preventive biomarker in lung cancer treatment and prognosis by regulating the AKT signaling pathway.

Keywords: Lung cancer; ribosomal protein L6 (RPL6); AKT pathway; cell apoptosis

Submitted Dec 27, 2021. Accepted for publication Feb 18, 2022.

doi: $10.21037 /$ jtd-22-116

View this article at: https://dx.doi.org/10.21037/jtd-22-116

\section{Introduction}

According to Global Cancer Statistics 2020 (GLOBOCAN 2020), 2.2 million new lung cancer cases and 1.8 million lung cancer deaths occurred in 2020 worldwide (1). Lung cancer is the leading cause of cancer morbidity and mortality in men, whereas in women, it ranks third for incidence after breast and colorectal cancer, and second for mortality after breast cancer (1). The traditional treatment methods for lung cancer include surgery, chemotherapy, radiotherapy, and multimodality therapies (2). Over the last decade, significant progress has been made in the treatment and diagnosis of lung cancer, such as curative intent, systemic targeted therapies, palliative care, and early diagnosis (3). However, the outcomes of lung cancer are still poor. The 5 -year survival rate was shown to be $<18 \%$, owing to poor prognosis (4). Therefore, it is of great 
significance to develop new treatment methods to improve the survival rate and quality of life of lung cancer patients.

Ribosomal protein L6 (RPL6), a highly conserved ribosomal protein, is an essential component of the $50 \mathrm{~S}$ subunit of ribosomes (5). RPL6 is located in the critical region that maps to chromosome $12 \mathrm{q} 24.1$ for Noonan syndrome (6). As reported by previous studies, RPL6 is involved in functional interaction with RbgA during ribosome assembly (7). RPL6 is recruited to DNA damage sites in a poly (ADP-ribose) polymerase-dependent manner and regulates DNA damage responses (8). Research has suggested that RPL6 can promote cell proliferation and cell cycle apoptosis though upregulating cyclin E in gastric cancer cells $(9,10)$. RPL6 was reported to regulate multidrug resistance in gastric cancer cells (11). In addition, a study suggested that RPL6 plays an important role in the development of drug resistance in K562/AO2 cells by regulating drug-induced apoptosis (12). These reports highlight the important role of RPL6 in solid tumors, but the detailed mechanism of the involvement of RPL6 in the development of lung cancer is still unknown. Since ribosome biogenesis plays a pivotal role in lung carcinogenesis (13), we intended to explore the significance of RPL6 in lung cancer in this research. We found that RPL6 was highly expressed in lung cancer tissue compared with paracancerous tissue, and the expression of RPL6 in 3 lung cancer cell lines was significantly higher than in normal cells. Furthermore, knockdown of RPL6 inhibited cell proliferation and migration and induced cell apoptosis in lung cancer cells, suggesting that it may act as a potential therapeutic biomarker in lung cancer treatment and prognosis by regulating the AKT signaling pathway. We present the following article in accordance with the MDAR reporting checklist (available at https://jtd.amegroups.com/ article/view/10.21037/jtd-22-116/rc).

\section{Methods}

\section{Clinical samples and immunobistochemistry}

A total of 26 lung cancer tissues and 15 normal tissues were obtained from the Department of Thoracic Surgery, ChinaJapan Friendship Hospital (Beijing, China) between June 2019 and May 2020. All patients were diagnosed with lung cancer by 2 pathologists. The study was approved by the human ethics and research ethics committees of ChinaJapan Friendship Hospital (approval No. 2019-95-K63-1) and written informed consent was obtained from all patients prior to participation. All procedures performed in this study involving human participants were in accordance with the Declaration of Helsinki (as revised in 2013).

The formalin-fixed paraffin-embedded lung cancer and normal samples were used to detect RPL6 expression by immunohistochemistry. Briefly, $4 \mu \mathrm{m}$ sections on slides were deparaffinized and rehydrated, followed by antigen retrieval. After washing, the slides were blocked by $5 \%$ bovine serum albumin (BSA), and then incubated with RPL6 antibody (Abcam, ab126100, 1:200) overnight at $4{ }^{\circ} \mathrm{C}$. Then, the slides were incubated with the secondary antibody for $1 \mathrm{~h}$. Lastly, the slides were stained by diaminobenzidine (DAB).

\section{Cell culture and RPL6 knockdown}

The lung cancer cell lines A549, H1975, and H1299 and the normal cell line BEAS-2B were obtained from American Type Culture Collection. All cells were maintained in RPMI-1640 medium (Gibco, Thermo Fisher Scientific, Waltham, MA, USA) with 10\% fetal bovine serum (Gibco, Thermo Fisher Scientific, USA) and $1 \%$ penicillin/streptomycin (Sigma, Merck KGaA, USA) in a humidified incubator containing $5 \% \mathrm{CO}_{2}$ at $37{ }^{\circ} \mathrm{C}$. RPL6 knockdown was performed in H1299 and H1975 cells using Lipofectamine RNAiMAX transfection reagent (Invitrogen, Thermo Fisher Scientific, Waltham, MA, USA). The siRNAs were purchased from GeneChem, and the sequences were as follows: siRPL6\#1: 5'-GCGCAAGAUUGAUCAGAAATT-3'; siRPL6\#2 : 5 ' - CUGCCAUGUAUUCCAGAAATT-3 '; siCtrl: 5'-UUCUCCGAACGUGUCACGU-3'.

\section{$R T-q P C R$ assay}

Total RNA was extracted from H1299 and H1975 cells using TRIzol reagent (Invitrogen). RNA concentration and purity was determined before RNA was reverse transcribed using qPCR RT master mix (Toyobo, Ltd. Osaka, Japan). RT-qPCR was conducted using the SYBR qPCR kit (TransGen Biotech) on the Real-time PCR System (BioRad). The RT-qPCR primers were as follows: RPL6-F: 5 '-CAGGAAGACATAAGGGAAAGCG-3', RPL6-R: 5 '-AGAGGGCAACCGTTCATAGC-3'; $\beta$-actin-F : 5 '-CATGTACGTTGCTATCCAGGC-3', $\beta$-actin-R: 5'-CTCCTTAATGTCACGCACGAT-3'.

\section{Western blotting}

Total proteins were extracted from cells lysed in RIPA buffer (Beyotime Biotechnology, Shanghai, China). The concentration of proteins was detected using the BCA kit 


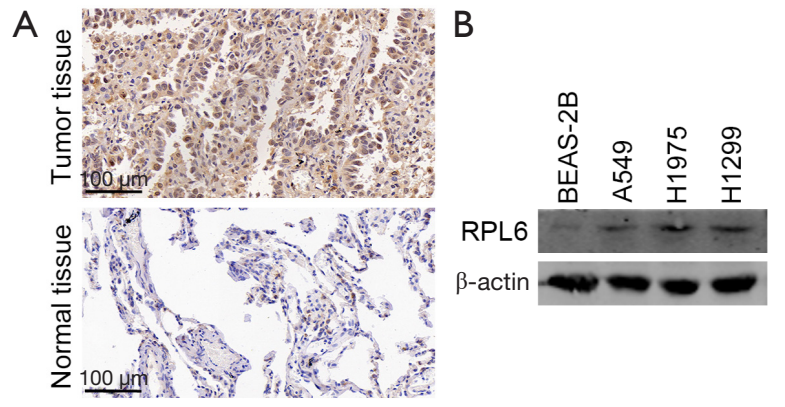

Figure 1 Upregulation of RPL6 in lung cancer. (A) Immunohistochemistry staining was performed to check RPL6 expression in lung cancer and paracancerous tissues. (B) Western blotting of RPL6 protein level in the 3 lung cancer cell lines. Bar, $100 \mu \mathrm{m}$. RPL6, ribosomal protein L6.

(Beyotime Biotechnology). SDS-PAGE was performed with $20 \mu \mathrm{g}$ total protein, which was then transferred to nitrocellulose membranes. Then, the membranes were blocked in 5\% non-fat milk at room temperature for $1 \mathrm{~h}$. Subsequently, the membranes were separately incubated with antibodies against RPL6 (Abcam, ab126100, 1:1,000), E-cadherin (Abcam, ab40772, 1:1,000), N-cadherin (Abcam, ab76011, 1:1,000), cleaved caspase-3 (Abcam, ab32042, 1:1,000), B-cell lymphoma 2 (Bcl-2) (Abcam, ab32124, 1:1,000), Bax (CST, \#2774, 1:1,000), PTEN (CST, \#9188, 1:1,000), phospho-AKT (CST, \#4060, 1:1,000), AKT (CST, \#9272, 1:1,000), P-S6 (CST, \#2211, 1:1,000), S6 (CST, \#2217, 1:1,000), and actin (CST, \#3700, 1:1,000) overnight at $4{ }^{\circ} \mathrm{C}$. Finally, secondary antibodies (Thermo Fisher Scientific) were added and incubated at room temperature for $2 \mathrm{~h}$. After washing 3 times by TBST, the bands were visualized using the enhanced chemiluminescence reagent (Cell Signaling Technology, Inc., Shanghai, China).

\section{Cell viability and colony formation assays}

Cell viability was assessed using the Cell Counting Kit8 (CCK-8, Beyotime Biotechnology) reagent. H1299 and H1975 cells $\left(2 \times 10^{3}\right)$ were added into a 96-well plate and cultured at $37^{\circ} \mathrm{C}$ for $24,48,72$, and $96 \mathrm{~h}$. A volume of $10 \mu \mathrm{L}$ CCK-8 reagent was added to each well and cultured at $37^{\circ} \mathrm{C}$ for $2 \mathrm{~h}$. The absorbance at $450 \mathrm{~nm}$ was measured by a microplate reader (Detie).

For the colony formation assay, H1299 and H1975 cells were added to a 6 -well plate at 500 cells per well. After 14 days of culture at $37{ }^{\circ} \mathrm{C}$, cells were fixed by $4 \%$ paraformaldehyde for $30 \mathrm{~min}$. Then, the cells were stained with $0.1 \%$ crystal violet for $20 \mathrm{~min}$.

\section{Cell apoptosis assay}

For the apoptosis assay, cells were collected and stained by the Annexin V-FITC apoptosis detection kit (Yeasen Biotechnology, Shanghai, China. Briefly, H1299 and H1975 cells transfected with siRPL6 or siCtrl were collected and suspended in binding buffer. Next, the cells were incubated with Annexin V for 15 $\min$. The cells were then washed and resuspended. Finally, the cells were incubated with $5 \mathrm{\mu l}$ propidium iodide for $15 \mathrm{~min}$ in the dark and subjected to flow cytometry for further analysis using a flow cytometer (Beckman).

\section{Transwell assay}

A total of $4.0 \times 10^{5}$ H1975 and H1299 cells transfected with siRPL6 or siCtrl were seeded into the upper chamber of the transwell and cultured in $200 \mu \mathrm{L}$ RPMI-1640 with no fetal bovine serum. Additionally, $500 \mu \mathrm{L}$ RPMI-1640 medium supplemented with $10 \%$ fetal bovine serum was added into the lower chamber. After being cultured at $37{ }^{\circ} \mathrm{C}$ for $24 \mathrm{~h}$, the cells were fixed with $4 \%$ paraformaldehyde and stained with $0.05 \%$ crystal violet.

\section{Statistical analysis}

The data are expressed as means \pm SD. Student's $t$-test was used to assess differences between 2 different groups. To analyze differences between more than 2 groups, one-way ANOVA followed by Tukey's post hoc test was performed. $\mathrm{P}<0.05$ was considered to indicate statistically significant differences.

\section{Results}

\section{RPL6 was upregulated in lung cancer}

The results of immunohistochemistry experiments showed that RPL6 was highly expressed in lung cancer tissue compared to paracancerous tissue (Figure $1 A$ and Table 1). Western blotting results indicated that RPL6 was highly expressed in all 3 lung cancer cell lines (Figure 1B). These results indicate that RPL6 is involved in lung cancer.

\section{RPL6 knockdown in lung cancer cell lines}

We used small-interfering RNAs to downregulate the expression of RPL6 in H1299 and H1975 cells. RT-qPCR (Figure 2A) and western blotting (Figure 2B) showed low 
Table 1 The expression of RPL6 in lung cancer and normal tissues

\begin{tabular}{lcccc}
\hline \multirow{2}{*}{ Type } & \multicolumn{2}{c}{ RPL6 expression } & \multirow{2}{*}{$\chi^{2}$} & P value \\
\cline { 2 - 3 } & Low & High & & \\
\hline Normal & 39 & 15 & 27.08 & $<0.001$ \\
Lung cancer & 12 & 42 & & \\
\hline
\end{tabular}

RPL6, ribosomal protein L6.
RPL6 expression levels in siRPL6-transfected H1299 and H1975 cells, confirming the knockdown.

\section{Knockdown of RPL6 significantly inbibited cell proliferation}

The viabilities of H1299 (Figure 3A) and H1975 cells (Figure 3B) transfected with siRPL6 were significantly decreased compared
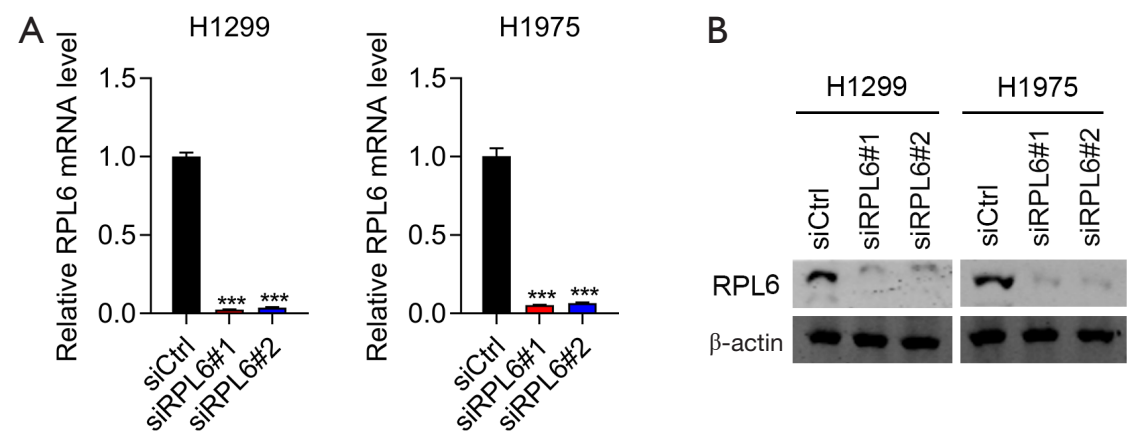

Figure 2 RPL6 knockdown in H1299 and H1975 cells. (A) RT-qPCR results of RPL6 mRNA levels in H1299 and H1975 cells transfected with siRPL6. (B) Western blotting results of RPL6 protein levels in H1299 and H1275 cells transfected with siRPL6. The loading control was GAPDH. ***, $\mathrm{P}<0.001$. RPL6, ribosomal protein L6.
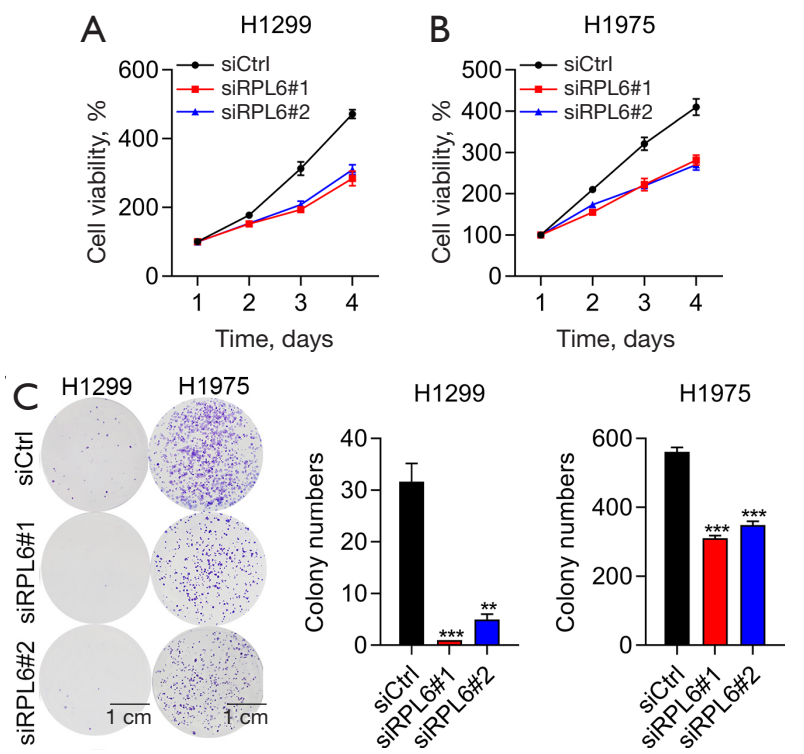

Figure 3 Knockdown of RPL6 inhibited cell proliferation. (A) CCK-8 results showing the viability of H1299 cells transfected with siCtrl, siRPL6\#1, and siRPL6\#2. (B) CCK-8 results showing the viability of H1975 cells transfected with siCtrl, siRPL6\#1, and siRPL6\#2. (C) Colony formation results of H1299 and H1975 cells transfected with siCtrl, siRPL6\#1, and siRPL6\#2. The cells were stained with $0.1 \%$ crystal violet. ${ }^{* *}, \mathrm{P}<0.01$; ***, $\mathrm{P}<0.001$. Bar, $1 \mathrm{~cm}$. RPL6, ribosomal protein L6. 

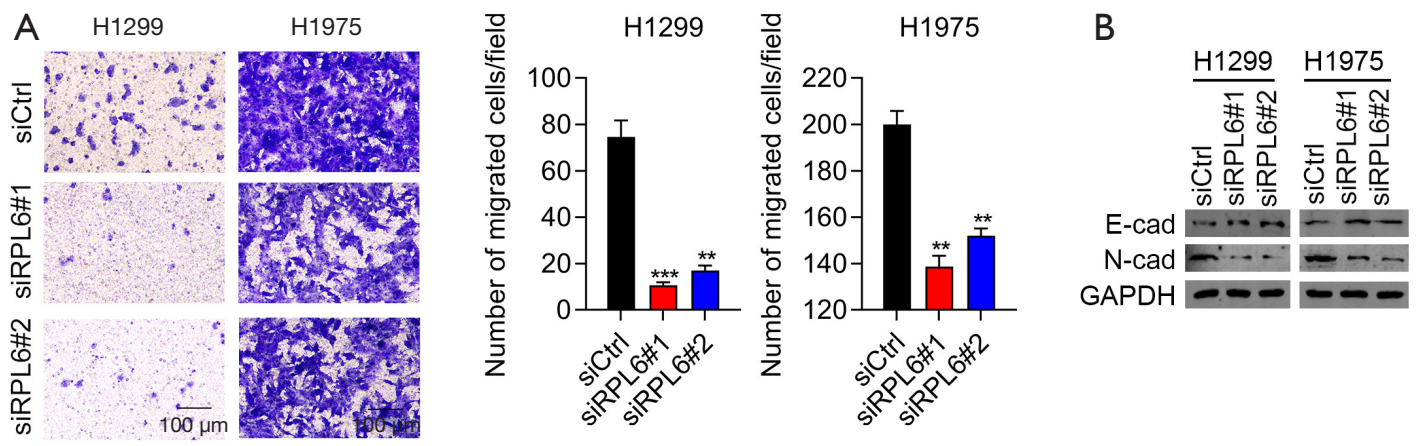

Figure 4 Knockdown of RPL6 inhibited cell migration. (A) Transwell results of H1299 and H1975 cells transfected with siCtrl, siRPL6\#1, and siRPL6\#2. The migrated cells were stained with $0.05 \%$ crystal violet. (B) Western blotting results of proteins related to migration in H1299 and H1975 cells transfected with siCtrl, siRPL6\#1, and siRPL6\#2. ** $\mathrm{P}<0.01$; ***, $\mathrm{P}<0.001$. Bar, 100 um. RPL6, ribosomal protein L6.
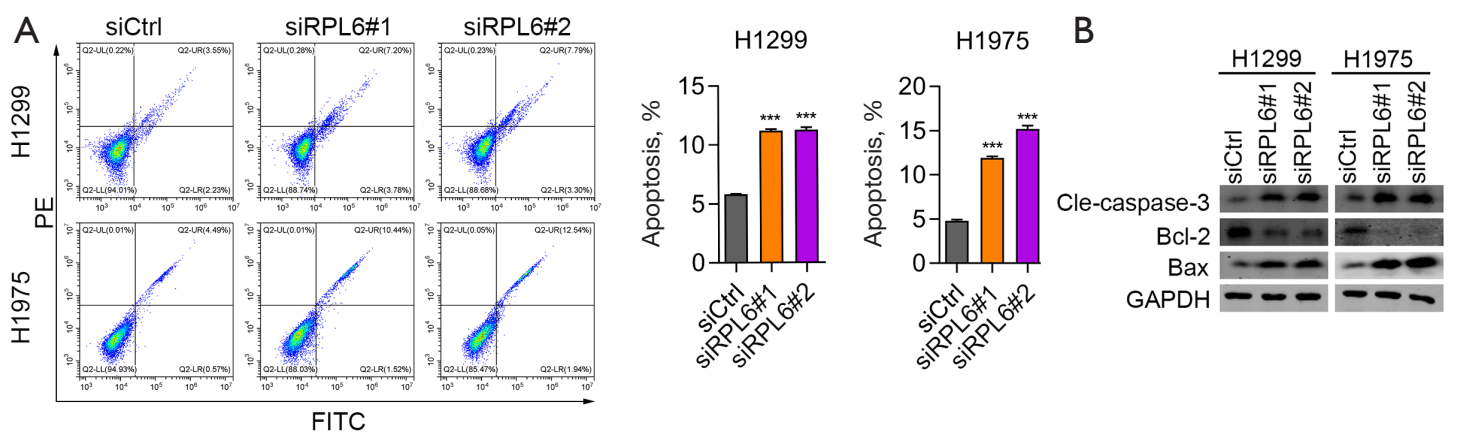

Figure 5 Knockdown of RPL6 induced cell apoptosis. (A) Flow cytometry results of H1299 and H1975 cells transfected with siCtrl, siRPL6\#1, and siRPL6\#2. (B) Western blotting results of proteins related to apoptosis in H1299 and H1975 cells transfected with siCtrl, siRPL6\#1, and siRPL6\#2. ***, $\mathrm{P}<0.001$. RPL6, ribosomal protein L6.

with cells transfected with siCtrl. The number of cell colonies formed by H1299 and H1975 cells transfected with siRPL6 were also decreased compared with siCtrl cells (Figure 3C).

\section{Knockdown of RPL6 inbibited cell migration}

The percentages of migrated H1299 and H1975 cells transfected with siRPL6 were significantly lower than cells transfected with siCtrl (Figure 4A). The protein level of $\mathrm{N}$-cadherin, which promotes cell migration, was also significantly decreased in H1299 and H1975 cells transfected with siRPL6 compared with siCtrl-transfected cells. In contrast, the protein expression of E-cadherin, which inhibits cell migration, was significantly increased (Figure 4B).

\section{Knockdown of RPL6 induced cell apoptosis}

Flow cytometry results showed that, compared with the
siCtrl group, the percentage of apoptotic cells in the 2 siRPL6 groups were increased by nearly double (Figure 5A). Western blotting results showed that cleaved caspase- 3 and Bax, which promote cell apoptosis, were upregulated in siRPL6-transfected H1299 and H1975 cells, while Bcl2, which inhibits apoptosis, had significantly decreased expression (Figure 5B). The results revealed the pro-apoptosis function of RPL6 knockdown in lung cancer cell lines.

\section{Knockdown of RPL6 inbibited the AKT signaling patbway}

Western blotting results showed that the p-AKT and p-S6 proteins in the AKT signaling pathway were significantly downregulated in siRPL6-transfected H1299 and H1975 cells, while phosphatase and tensin homolog (PTEN) was significantly upregulated (Figure 6). The results showed that downregulated RPL6 inhibits cell proliferation and migration and induces cellular apoptosis through the AKT 


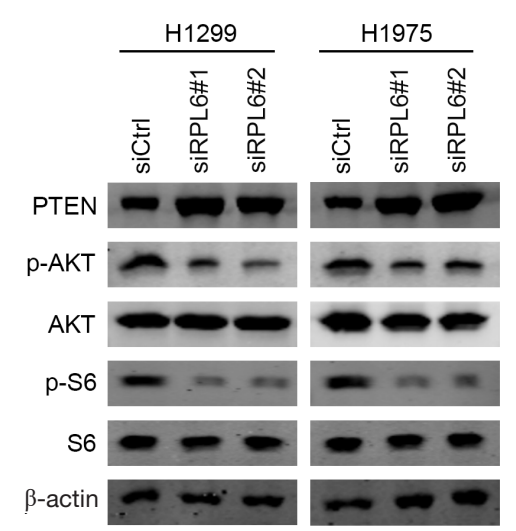

Figure 6 Knockdown of RPL6 inhibits the AKT signaling pathway. Western blotting results of proteins related to the AKT signaling pathway in H1299 and H1975 cells transfected with siCtrl, siRPL6\#1, and siRPL6\#2. RPL6, ribosomal protein L6.

signaling pathway.

\section{Discussion}

Lung cancer is the leading cause of cancer-related deaths worldwide. The underlying molecular mechanisms need to be better understood to offer improved treatment for lung cancer. RPL6 is reported to participate in the cellular response to multidrug resistance (11) and acts as a critical regulator of cell growth, the cell cycle in solid tumors $(9,12)$, and the DNA damage response (8). Nevertheless, the potential functions and underlying mechanism of RPL6 in lung cancer development remain unclear. In this study, we showed that RPL6 was highly expressed in lung cancer and that its knockdown could suppress the proliferation and migration of lung cancer cells. At the same time, western blotting results showed that the protein level of E-cadherin was increased while $\mathrm{N}$-cadherin was significantly decreased in lung cancer cells after RPL6 knockdown. Cadherins are calcium-dependent cell adhesion proteins (14). They preferentially interact with themselves in a homophilic manner to facilitate cell adhesion. Cadherins may therefore contribute to the sorting of heterogeneous cell types. E-cadherin, with locus on chromosome 16, is involved in mechanisms regulating cell-cell adhesion, mobility, and the proliferation of epithelial cells as a tumor suppressor (14). $\mathrm{N}$-cadherin is mapped to chromosome 18 and has important roles in the early stages of neural development, including in the proliferation and differentiation of neural progenitor cells, thereby serving as an oncogene (15). Downregulation of E-cadherin and upregulation of $\mathrm{N}$-cadherin are the markers of epithelial to mesenchymal transition (EMT), which plays an essential role in regulating cancer metastasis. Additionally, metastasis is the major risk factors for the death of lung cancer patients. Thus, we proposed that RPL6 might be involved in the patient risk stratification and local regional metastasis in lung cancer.

Furthermore, flow cytometry results showed that RPL6 knockdown-induced apoptosis of lung cancer cells and the protein levels of cleaved caspase- 3 and Bax were increased, while Bcl-2 expression decreased significantly. Apoptosis is orchestrated by caspases, a family of cysteine proteases that cleave their substrates on the carboxy-terminal side of specific aspartic acid residues. Therefore, the detection of cleaved caspase- 3 is considered a reliable marker for cells that are dying or have died by apoptosis (16). Bcl-2 is an anti-apoptotic gene which is anti-proliferative by facilitating G0 (17). $\mathrm{BAD}$ is a BH3-only member of the Bcl-2 family that possesses important regulatory functions in apoptosis, and antagonizes the cell cycle and anti-apoptotic functions of Bcl-2 (18).

Lastly, we detected the expression levels of proteins involved in the AKT signaling pathway and found that p-AKT and p-S6 were significantly downregulated while PTEN was significantly upregulated in RPL6knockdown cells. The AKT signaling pathway is a critical signaling pathway in the cellular processes of cancers and is closely related to cell apoptosis, migration, invasion, and differentiation (19). AKT, also known as protein kinase $\mathrm{B}(\mathrm{PKB})$, is an attractive therapeutic target in cancer as it inactivates pro-apoptotic proteins such as $\mathrm{BAD}$ to block apoptosis, and p-AKT is the activated form of AKT (20). PTEN is a notable tumor suppressor and can negatively regulate the PTEN/PI3K/AKT signaling pathway through dephosphorylation of AKT (21). p-S6 is a downstream molecule in the mTOR pathway, and its expression level is associated with the overall survival of some solid cancers, thereby having the potential to be a prognostic factor (22). These results suggest that RPL6 likely functions as an oncogene by activating the AKT signaling pathway in lung cancer cell proliferation and migration. AKT/mTOR signaling is involved in regulating the activity of other signaling pathways, such as GSK-3 $\beta / \beta$-catenin, MDM2/ P53, autophagy, and glycolysis $(23,24)$. So RPL6 might also act as the master modulator for these signals.

There were some limitations in this study. (I) We did not investigate upstream non-coding RNAs which might 
regulate RPL6 expression, including hsa_circ_0006848/ hsa_miR-329-5p axis (25), hsa-miR-5690, hsa-miR-7852$3 \mathrm{p}$, hsa-miR-4698 and hsa-miR-4311, which were analyzed from TargetScan. (II) Rescue experiments were needed to explore the contribution of AKT/mTOR signaling pathway to the oncogenic function of RPL6. In the future, we will perform intensive experiments to illustrate the upstream and downstream singaling of RPL6 in lung cancer.

\section{Conclusions}

We demonstrated that RPL6 functions as an oncogene in lung cancer via regulating the AKT signaling pathway. Upregulation of RPL6 expression was observed in lung cancer samples and its downregulation suppressed the proliferation and invasion of H1299 and H1975 cells. Downregulation of RPL6 expression also suppressed the migration of H1299 and H1975 cells, decreased the protein levels of oncogenes related to cell migration such as $\mathrm{N}$-cadherin, while at the same time, increased the protein level of anti-tumor genes such as E-cadherin. Furthermore, RPL6 knockdown induced cell apoptosis and promoted the expression of apoptosis-related proteins such as cleaved caspase- 3 and Bax. The p-AKT and p-S6 proteins related to the AKT signaling pathway were significantly decreased in RPL6 knockdown cells. Therefore, our study sheds light on the function of RPL6 in lung cancer progression via the AKT signaling pathway. The findings in this study suggested that overexpression of RPL6 might serve as a diagnostic and therapeutic target for lung cancer. In the future, intensive studies should be carried out on the drugs which can inhibit the function of RPL6 that may benefit for lung cancer patients.

\section{Acknowledgments}

Funding: This study was supported by the Project of Disciplines Construction of State Key Clinical Department [No. (2011)873], and Elite Medical Professionals Project of China-Japan Friendship Hospital (No. ZRJY2021-GG07).

\section{Footnote}

Reporting Checklist: The authors have completed the MDAR reporting checklist. Available at https://jtd.amegroups.com/ article/view/10.21037/jtd-22-116/rc

Data Sharing Statement: Available at https://jtd.amegroups. com/article/view/10.21037/jtd-22-116/dss

Conflicts of Interest: All authors have completed the ICMJE uniform disclosure form (available at https://jtd.amegroups. com/article/view/10.21037/jtd-22-116/coif). The authors have no conflicts of interest to declare.

Ethical Statement: The authors are accountable for all aspects of the work in ensuring that questions related to the accuracy or integrity of any part of the work are appropriately investigated and resolved. The study was approved by the human ethics and research ethics committees of China-Japan Friendship Hospital (approval No. 2019-95-K63-1). Written informed consent was obtained from each patient. All procedures performed in this study involving human participants were in accordance with the Declaration of Helsinki (as revised in 2013).

Open Access Statement: This is an Open Access article distributed in accordance with the Creative Commons Attribution-NonCommercial-NoDerivs 4.0 International License (CC BY-NC-ND 4.0), which permits the noncommercial replication and distribution of the article with the strict proviso that no changes or edits are made and the original work is properly cited (including links to both the formal publication through the relevant DOI and the license). See: https://creativecommons.org/licenses/by-nc-nd/4.0/.

\section{References}

1. Sung H, Ferlay J, Siegel RL, et al. Global Cancer Statistics 2020: GLOBOCAN Estimates of Incidence and Mortality Worldwide for 36 Cancers in 185 Countries. CA Cancer J Clin 2021;71:209-49.

2. Kakimi K, Matsushita H, Murakawa T, et al. $\gamma \delta \mathrm{T}$ cell therapy for the treatment of non-small cell lung cancer. Transl Lung Cancer Res 2014;3:23-33.

3. Jones GS, Baldwin DR. Recent advances in the management of lung cancer. Clin Med (Lond) 2018;18:s41-6.

4. Zappa C, Mousa SA. Non-small cell lung cancer: current treatment and future advances. Transl Lung Cancer Res 2016;5:288-300.

5. Shigeno $Y$, Uchiumi T, Nomura T. Involvement of ribosomal protein L6 in assembly of functional 50S ribosomal subunit in Escherichia coli cells. Biochem Biophys Res Commun 2016;473:237-42.

6. Kenmochi N, Yoshihama M, Higa S, et al. The human 
ribosomal protein L6 gene in a critical region for Noonan syndrome. J Hum Genet 2000;45:290-3.

7. Gulati M, Jain N, Davis JH, et al. Functional interaction between ribosomal protein L6 and RbgA during ribosome assembly. PLoS Genet 2014;10:e1004694.

8. Yang C, Zang W, Ji Y, et al. Ribosomal protein L6 (RPL6) is recruited to DNA damage sites in a poly(ADP-ribose) polymerase-dependent manner and regulates the DNA damage response. J Biol Chem 2019;294:2827-38.

9. Gou Y, Shi Y, Zhang Y, et al. Ribosomal protein L6 promotes growth and cell cycle progression through upregulating cyclin E in gastric cancer cells. Biochem Biophys Res Commun 2010;393:788-93.

10. Wu Q, Gou Y, Wang Q, et al. Downregulation of RPL6 by siRNA inhibits proliferation and cell cycle progression of human gastric cancer cell lines. PLoS One 2011;6:e26401.

11. Du J, Shi Y, Pan Y, et al. Regulation of multidrug resistance by ribosomal protein 16 in gastric cancer cells. Cancer Biol Ther 2005;4:242-7.

12. Chen H, Xie ZX, Jiang H, et al. Effect of ribosomal protein L6 on drug resistance and apoptosis in K562/ A02 cells. Zhongguo Shi Yan Xue Ye Xue Za Zhi 2007; 15:292-5.

13. Wang X, Zhang H, Sapio R, et al. SOD1 regulates ribosome biogenesis in KRAS mutant non-small cell lung cancer. Nat Commun 2021;12:2259.

14. Meigs TE, Fedor-Chaiken M, Kaplan DD, et al. Galpha12 and Galpha13 negatively regulate the adhesive functions of cadherin. J Biol Chem 2002;277:24594-600.

15. Hatta K, Takagi S, Fujisawa H, et al. Spatial and temporal expression pattern of $\mathrm{N}$-cadherin cell adhesion molecules correlated with morphogenetic processes of chicken

Cite this article as: Zhang J, Ma Q, Han Y, Wen H, Zhang Z, Hao Y, Xiao F, Liang C. Downregulated RPL6 inhibits lung cancer cell proliferation and migration and promotes cell apoptosis by regulating the AKT signaling pathway. J Thorac Dis 2022;14(2):507-514. doi: 10.21037/jtd-22-116 embryos. Dev Biol 1987;120:215-27.

16. Crowley LC, Waterhouse NJ. Detecting Cleaved Caspase-3 in Apoptotic Cells by Flow Cytometry. Cold Spring Harb Protoc 2016. doi: 10.1101/pdb.prot087312.

17. Zinkel S, Gross A, Yang E. BCL2 family in DNA damage and cell cycle control. Cell Death Differ 2006;13:1351-9.

18. Bui NL, Pandey V, Zhu T, et al. Bad phosphorylation as a target of inhibition in oncology. Cancer Lett 2018;415:177-86.

19. Gao N, Zhang Z, Jiang BH, et al. Role of PI3K/ AKT/mTOR signaling in the cell cycle progression of human prostate cancer. Biochem Biophys Res Commun 2003;310:1124-32.

20. Shariati M, Meric-Bernstam F. Targeting AKT for cancer therapy. Expert Opin Investig Drugs 2019;28:977-88.

21. Xun $\mathrm{G}, \mathrm{Hu}$ W, Li B. PTEN loss promotes oncogenic function of STMN1 via PI3K/AKT pathway in lung cancer. Sci Rep 2021;11:14318.

22. Zheng Z, Zheng Y, Zhang M, et al. Reciprocal expression of p-AMPKa and p-S6 is strongly associated with the prognosis of gastric cancer. Tumour Biol 2016;37:4803-11.

23. Manning BD, Toker A. AKT/PKB Signaling: Navigating the Network. Cell 2017;169:381-405.

24. Manning BD, Cantley LC. AKT/PKB signaling: navigating downstream. Cell 2007;129:1261-74.

25. Lu J, Zhang PY, Xie JW, et al. Circular RNA hsa_ circ_0006848 Related to Ribosomal Protein L6 Acts as a Novel Biomarker for Early Gastric Cancer. Dis Markers 2019;2019:3863458.

(English Language Editor: C. Betlazar-Maseh) 